[Note de l'auteur: La version publiée de cet article, dont je n'ai pas eu l'occasion de vérifier les épreuves, contient de nombreuses erreurs de formatage affectant notamment les citations en vers. Le présent fichier donne une version correctement formatée, qui correspond exactement au texte et à la mise en page de la version publiée.]

\title{
Madame Deshoulières, ou la satire au féminin
}

\author{
Pour François Rigolot
}

Deshoulieres (Antoinette de La Garde). De toutes les dames françaises qui ont cultivé la poésie, c'est celle qui a le plus réussi, puisque c'est celle dont on a retenu le plus de vers. C'est dommage qu'elle soit l'auteur du mauvais sonnet contre l'admirable Phèdre de Racine. Ce sonnet ne fut bien reçu du public que parce qu'il était satirique. N'est-ce pas assez que les femmes soient jalouses en amour ? faut-il encore qu'elles le soient en belles-lettres? Une femme satirique ressemble à Méduse et à Scylla, deux beautés changées en monstres. Morte en $1694^{1}$.

Le présent article vise à affronter cet aspect «monstrueux» de Mme Deshoulières en suivant les avatars de la satire dans son œuvre poétique. Car le penchant satirique déploré par Voltaire ne se limite pas au sonnet (d'ailleurs anonyme) sur Phèdre et aux éphémères cabales littéraires; plutôt qu'un égarement momentané, il représente une inspiration constante qui traverse l'œuvre entière et s'exprime d'une façon tantôt plus discrète, tantôt plus éclatante. Ce talent pour la satire est en effet une des qualités que Mlle Lhéritier met en valeur dans son hommage funèbre à Mme Deshoulières. Celle-ci «avoit brillé dans le monde par tant de caracteres diferens, qu'on avoit peine à déterminer sous lequel on devoit la ranger » aux Champs-Élysées : parmi les Belles ? les Enjouées ? les Joueuses? Embarrassé, Pluton remarque :

qu'à plus juste titre les Satyriques agreables se récriroient qu'elle devoit être de leur Troupe, elle qui par les traits fins d'une Satyre toûjours vive, sans blesser jamais personne, avoit avec tant de délicatesse \& d'esprit, censuré les défauts des hommes \& les travers du siecle ${ }^{2}$.

${ }^{1}$ Voltaire, Le Siècle de Louis XIV, dans Euvres historiques, éd. René Pomeau, Paris, Gallimard, 1957, p. 1156.

2 Le Parnasse reconnoissant, on le Triomphe de Mme Des-Houlières, dans CEurres meslées, Paris, Jean Guignard, 1696, p. 407. - En 1692, Callières avait terminé son manuel de la « raillerie » sur l'éloge du «beau \& fecond genie » de Mme Deshoulières : celle-ci ne célèbre pas seulement Louis XIV, « elle sçait aussi railler \& badiner finement \& agréablement quand il luy plait, \& elle nous a donné des ouvrages qui nous font connoître qu'elle excelle en ce genre, ainsi qu'en plusieurs autres especes de poisië [sic] 》 (Des bons mots et des bons contes, Paris, Barbin, 1692, pp. 341-342). 
Mais ne faudrait-il pas la placer plutôt avec les Savantes et les Spirituelles? Finalement, c'est Apollon qui règle la question en installant Mme Deshoulières sur le Parnasse où elle trônera désormais en Dixième Muse.

À l'instar de Pluton, le lecteur moderne qui rencontre l'œuvre de Mme Deshoulières peut se sentir perplexe devant la coexistence de «tant de caracteres diferens ». Les deux récentes éditions de ses poésies complètes ${ }^{3}$ mettent en évidence toute la diversité de ce corpus que la postérité avait peu à peu réduit aux fameuses idylles, avant de l'oublier complètement. Cette heureuse renaissance éditoriale va de pair avec un regain d'intérêt critique qui, à vrai dire, s'était fait attendre, car en dépit de tant de travaux novateurs sur les femmes écrivains du XVII siècle, Mme Deshoulières restait bizarrement négligée et ne suscitait qu'un fort petit nombre d'études. Ce n'est que depuis une dizaine d'années qu'elle commence à sortir de l'obscurité et à s'approcher de nouveau de la place qu'elle occupait jadis sur le Parnasse français, réhabilitation due en partie aux efforts de chercheurs américains 4 .

Parallèlement s'est poursuivie de divers côtés la réflexion sur le statut des «femmes satiriques", à commencer par cette question : existe-t-il vraiment de ces «monstres »? À l'exception de la mystérieuse Sulpicia ${ }^{5}$, la tradition de la satire en vers depuis l'Antiquité semble en effet presque exclusivement masculine; si les études sur le discours satirique abordent les femmes, c'est en général en tant qu'objets et cibles, et rarement en tant que sujets parlants et actifs. En 1994, Dustin Griffin désigna cette problématique comme un des desiderata de la satirologie :

Why, for example, have women writers been excluded from the canon of satire? Have they (like women elsewhere) been excluded by male readers, or have they excluded themselves? Whichever the case, the organization of culture has made it difficult for women to write and publish satire. [...] To discover how women writers sought to evade or overcome such discouragements might not only expand our sense of the range of satire from Aphra Behn to Jane Austen but might also enhance our sense of how satire functions within a culture ${ }^{6}$.

3 Antoinette Des Houlières, L'Enchantement des chagrins : poésies complètes, éd. Catherine HémonFabre et Pierre-Eugène Leroy, Paris, Bartillat, 2005; Madame Deshoulières, Poésies, éd. Sophie Tonolo, Paris, Classiques Garnier, 2010.

4 Pour la fortune de l'œuvre et les développements de la critique, voir l'introduction de Sophie Tonolo à son édition, ainsi que mon article "Verse and Versatility: The Poetry of Antoinette Deshoulières", dans Teaching Seventeenth-and Eighteenth-Century French Women Writers, Faith E. Beasley (ed.), New York, MLA, 2011, pp. 242-249.

${ }^{5}$ Femme poète de la fin du $1^{\text {er }}$ siècle, épouse vertueuse d'un certain Calenus, à laquelle a longtemps été attribué un poème de 70 hexamètres contre l'empereur Domitien. Redécouvert en 1493, le texte fut parfois publié à la suite de Pétrone ou de Juvénal ; en 1658, Marolles le traduit dans le cadre de son édition bilingue des Satires de Juvénal et Perse. Aujourd'hui, cette satire est généralement considérée comme apocryphe; seuls deux vers authentiques de Sulpicia semblent avoir survécu. "She is a lost Roman satirist, the only woman writer associated with any comic genre in antiquity as far as I know. » (Amy Richlin, «Sulpicia the Satirist », Classical World, no 86, 1992-93, p. 126).

6 Satire: A Critical Reintroduction, Lexington, University Press of Kentucky, 1994, pp. 189-190. Cf. Charles A. Knight, The Literature of Satire, Cambridge, Cambridge University Press, 2004, pp. 6-7 ; Claudia Kairoff, "Gendering Satire: Behn to Burney», dans A Companion to Satire: Ancient and Modern, Ruben Quintero (ed), Oxford, Blackwell, 2007, pp. 276-292. 
Appliquée par Griffin au domaine anglais, cette interrogation est bien entendu tout aussi féconde pour la France du XVII ${ }^{\mathrm{e}}$ siècle. Pascal Debailly souligne ainsi l'apparente incompatibilité entre la satire et le public féminin :

Peu friandes d'épopées guerrières, les femmes n'aiment pas lire non plus des satires, dont elles sont le plus souvent la cible et le tiers exclu. Virile, misogyne, souvent obscène, cette forme d'écriture contredit trop l'idéal de bienséance et de civilité qui se développe au XVII et au XVIII ${ }^{\mathrm{e}}$ siècle, sous l'influence déterminante des femmes. Leur jugement est en tout cas largement responsable de son déclin et de sa disparition ${ }^{7}$.

Si les femmes n'aiment pas lire des satires, comment a fortiori oseraient-elles en composer? Et pourtant, nous avons vu Mlle Lhéritier ranger sa consœur parmi les «Satyriques agreables» et distinguer dans sa poésie «les traits fins d'une Satyre toûjours vive ».

Je me propose donc de retracer ici quelques-uns de ces traits satiriques, aussi bien pour illustrer les possibilités et modalités d'une certaine satire féminine à l'âge classique que pour faire ressortir une dimension caractéristique mais peu étudiée de l'œuvre de Mme Deshoulières. Dans les limites de cet article, je dois me borner à un rapide parcours (à peu près chronologique) qui tentera de poser quelques jalons, en mettant l'accent sur la représentation poétique de la parole moqueuse, critique ou agressive, celle des femmes comme celle des hommes.

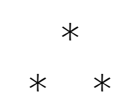

La double face de la satire apparaît dès les premiers textes de Mme Deshoulières, publiés dans le Recueil des portraits et éloges de 1659. Elle y fait le portrait de Lignières, poète libertin et auteur de brocards (contre Chapelain et, plus tard, Boileau, entre autres), qu'elle présente comme un « aimable imposteur» :

Il paraît ingénu, bon et sans artifice,

Mais son air est trompeur, il a de la malice,

Il aime la satire, et croit qu'il est permis

De railler fortement de ses meilleurs Amis,

D'aimer en divers lieux, de faire des promesses,

De signer des contrats pour fourber ses maitresses ${ }^{8}$.

Le goût de la satire est ici associé, non pas avec la franchise mais avec la perfidie et, plus précisément, l'inconstance et l'infidélité amoureuse - défaut masculin que ne cesse de vilipender Mme Deshoulières tout au long de sa vie. Le satirique Lignières est un véritable Don Juan ${ }^{9}$ qui se croit tout permis et ne tient pas sa parole ; le por-

${ }^{7}$ La Muse indignée, t. I, Paris, Classiques Garnier, 2012, p. 27.

8 Poésies, éd. Sophie Tonolo, Paris, Classiques Garnier, 2012, p. 91. Toutes mes citations de Mme Deshoulières renvoient à cette édition; le numéro de page sera indiqué entre parenthèses après la citation.

9 Voir Jacqueline Plantié, La Mode du portrait littéraire en France (1641-1681), Paris, Champion, 1994, pp. 399-403. 
trait se termine en effet sur l'évocation de son impiété : «Quoiqu'il raille souvent des articles de Foi, / Je crois qu'il est autant Catholique que moi » (p. 94). Dans sa délicieuse ambiguité ironique, ce dernier vers signale cependant que le portrait lui-même relève de la raillerie amicale et complice, que l'auteur justifie au nom de son «art de peindre au naturel», opposé à l'artifice masculin : «Je dis naïvement et le bien et le mal» (p. 92).

Aussi Lignières reconnait-il que sur ce chapitre de la foi Mme Deshoulières le « raille de bonne grace ${ }^{10} »$. Dans les portraits qu'il compose d'elle, il ne manque pas de relever son humeur railleuse :

Elle a l'Esprit tourné vers la galanterie ;

Elle n'aime pas moins la fine raillerie,

L'agreable Satyre ; \& pour les médisans,

Ils luy sont devenus fâcheux $\&$ déplaisans ${ }^{11}$.

Alors que le poète « aime la satire » et « raille fortement » de ses amis, la poétesse aime «la fine raillerie» et «l'agreable Satyre»: distinctions fines mais significatives, renforcées aussitôt par la condamnation des "médisans", qu'il ne faut donc surtout pas confondre avec les railleurs plaisants.

Ces vers reflètent les débats mondains sur l'idéal complexe de la « raillerie » et ses rapports difficiles avec la satire et la médisance, tels qu'on les lit notamment chez Mlle de Scudéry. La romancière tend à prôner une distinction radicale entre la raillerie et la satire : «il faut qu'il y ait un si grand intervalle, entre la raillerie, et la satire, qu'on ne puisse jamais prendre l'une pour l'autre ${ }^{12} »$. D'autre part, comme le montre l'exemple de Mlle Lhéritier, certaines femmes écrivains refusent d'abandonner la notion de «satire » et essaieront plutôt de définir et défendre une manière " agréable » de la pratiquer. Mme Deshoulières, quant à elle, évite prudemment cette appellation générique suspecte (aucun de ses poèmes n'est intitulé « satire ») tout en s'appropriant à sa façon les principaux éléments de l'écriture satirique traditionnelle. Sa stratégie prolonge ainsi celle de ses prédécesseurs masculins, les poètes mondains du milieu du siècle étudiés par Alain Génetiot: « la véritable satire [...] est absente de notre corpus de genres mondains. Par contre, la fonction satirique (se moquer) est omniprésente, et la raillerie, la moquerie trouvent de nouvelles formes pour se faire jour ${ }^{13}$. »

Chez Mme Deshoulières, l'expérimentation avec les divers registres satiriques passe d'abord par l'emploi d'une persona animalière. Le premier poème qu'elle donne

10 Recuieil des Portraits et éloges en vers et en prose dedié à son Altesse Royalle Mademoiselle, Paris, Sercy et Barbin, 1659, p. 900.

${ }^{11}$ Ibid., p. 380. Cf. aussi le «Portrait d'Amarante» que Lignières adresse à Mme Deshoulières et dont elle semble être à la fois la destinataire et le modèle : «Sa Satyre n'est point grossiere, / Elle dit nos defauts d'une douce maniere ; / Sa raillerie est un poison charmant, / On l'écoute agreablement» (p. 556).

12 «De la raillerie », dans "De l'air galant» et autres conversations, éd. Delphine Denis, Paris, Champion, 1998, p. 110. Cette conversation parut en 1653 dans le tome IX d'Artamène ou le Grand Cyrus et fut reprise en 1680 dans les Conversations sur divers sujets.

${ }^{13}$ Alain Génetiot, Les Genres lyriques mondains (1630-1660) : étude des poésies de Voiture, Vion d'Alibray, Sarasin et Scarron, Genève, Droz, 1990, p. 140 ; voir aussi pp. 145-150 sur la « fine raillerie ». 
au Mercure galant (revue qui sera son principal débouché de publication, de 1672 à 1694) est une «Lettre de Gas, épagneul de Madame Deshoulières, à Monsieur le Comte de L.T. », qui s'ouvre sur cette attaque brusque:

Pour vous marquer mon courroux,

J'ai mis la plume à la patte ;

Il est temps que contre vous

Toute ma colère éclate. (p. 95)

Pour son début dans le Mercure, Mme Deshoulières présente une variation burlesque du Facit indignatio versum de Juvénal, dont la voix irritée et agressive est ici prêtée à... un épagneul, fier de son "naturel incommode et farouche ». Il s'agit pourtant bien d'une pièce galante, car Gas écrit, non pour dénoncer la décadence des mœurs, mais pour défendre mordicus sa place sur le lit de sa maittresse contre les assauts des prétendants ${ }^{14}$.

Le mordant du fidèle toutou ne se limite cependant pas à la jalouse défense de ses intérêts personnels; dans un autre poème - "Apothéose de Gas mon chien, à Iris »-Mme Deshoulières lui confère la fonction de censeur public de la vie littéraire. Sur le conseil de «la badine Érato» et contre l'opposition de «Messieurs les Auteurs », Apollon immortalise le « téméraire chien » et en fait le gardien des Lettres:

Déchire à belles dents ceux dont la folle audace

De mes doctes chansons croit emporter le prix,

Et pour ces demi-beaux esprits

Sois le Cerbère du Parnasse. (p. 375)

Voilà que Gas barre le passage aux mauvais poètes et « $\mathrm{A}$ ceux dont tous les Hémistiches / Sont pleins de médisance, ou pleins de mots impurs». Le texte se termine par les portraits de trois auteurs qui se trouvent ainsi exclus du Parnasse, «le vieux Lisis » (un ami de Malherbe), «le pétulant Albin» (un docte aristotélicien) et «l'ignorant Timandre» (buveur et joueur) : aux lecteurs d'identifier les cibles de ces coups de patte.

Sur ordre d'Apollon, l'épagneul tient ainsi le rôle que Boileau - que ses adversaires nommaient le «cynique Despréaux » - s'arroge lui-même depuis quelques années ${ }^{15}$. Vision à la fois parodique et utopique, cette apothéose de l'épagneul imagine

${ }^{14}$ Cette lettre inaugure une série de correspondances galantes entre animaux domestiques, dans lesquelles Mme Deshoulières donne le rôle central à sa chatte Grisette. Dans une de ses lettres à Cochon (chien du maréchal de Vivonne), Grisette établit la distinction suivante entre la «Race cynique » et sa propre espèce : «Vous ne mordez pas mal. Pour vous rien n’est sacré. / Devant vous rien ne trouve grâce. / Vous déchirez tout [...] Si vous avez de bonnes dents / Nous n'avons pas mauvaise griffe. / Cependant, comme j'aime à n'en jamais user [...]»; elle se prévaut de sa «modeste gueule » dont «Jamais cris aigus, scandaleux, / Ne sont sortis » (Poésies, pp. 401-403).

15 Boileau lui-même endosse ce masque canin : «Mais tout fat me déplait, et me blesse les yeux; / Je le poursuis partout, comme un chien fait sa proie, / Et ne le sens jamais qu'aussitôt je n'aboie.» («Satire VII», dans Satires, Épittres, Art poétique, éd. Jean-Pierre Collinet, Paris, Gallimard, 1985, p. 95). Voici Gas : «Il démêle un sot de cent pas, / Le poursuit, l'aboie, et le pille » (Poésies, p. 374). 
l'institution d'un contre-régent du Parnasse, protecteur docile de sa maitresse et des muses. Pendant que le brave gars se charge de pourfendre les méchants auteurs, les dames peuvent jouir « du repos et de l'ombre $^{16} »$.

L' «Apothéose de Gas » suggère que Mme Deshoulières, au début des années 1670, était tentée de concurrencer Boileau et se lancer à son tour dans la satire littéraire, de façon certes moins directe et brutale. Elle finit cependant par reculer; le texte resta en fait inachevé et ne fut imprimé qu'après sa mort ${ }^{17}$. Dans la suite de sa carrière, elle n'hésita pas à s'attaquer nommément à d'autres écrivains : outre le sonnet notoire sur Phèdre, les éditions posthumes de ses œuvres contiennent plusieurs poèmes - sonnets, épigrammes, chansons, parodies - qui raillent Pradon et Bouhours, Saint-Gilles et Du Perrier, ou encore l'académicien Testu. Mais de son vivant ces textes ne circulaient que sous forme manuscrite et leur attribution est parfois tardive ou incertaine. Cette manière discrète voire secrète de pratiquer la satire littéraire (y compris ad hominem) et d'intervenir dans les querelles trouve un écho dans la caricature que fait Boileau dans sa "Satire $\mathrm{X}$ » de la "précieuse », qu'on a souvent identifiée à Mme Deshoulières: c'est «chez elle» que «la belle $[\ldots]$ fait la guerre» au "mauvais goût public », soutenant les «fades auteurs» et riant de leurs rivaux ${ }^{18}$.

Plutôt que de s'exposer sur le champ de bataille de la satire littéraire et de s'attaquer ouvertement aux individus, Mme Deshoulières privilégie en définitive la satire morale et sociale à caractère général. Celle-ci est plus «innocente» dans la mesure où elle ne blesse ou diffame personne en particulier, mais aussi plus radicale voire subversive par sa dénonciation de la corruption du «siècle» ou $\mathrm{du}$ «genre humain» tout entiers. Aussi l'auteur prend-elle soin de masquer son audace et de tempérer son indignation, tantôt en adoptant un ton enjoué, tantôt en délaissant le badinage pour la gravité du discours moraliste et philosophique ${ }^{19}$. Dans aucun cas, répétons-le, ces poèmes ne se présentent explicitement comme des «satires»: quand l'envie lui prend de moquer les ridicules ou de fulminer contre les vices (c'est-à-dire pas toujours, mais assez souvent), Mme Deshoulières a recours à un des nombreux autres genres poétiques que la tradition antique et la mode mondaine mettent à sa disposition: idylles et odes, épitres et chansons, ballades et rondeaux. L'élan satirique est contrôlé et pour ainsi dire civilisé par son insertion dans un cadre générique en apparence inoffensif et bienséant ${ }^{20}$.

${ }^{16}$ Ibid. Sur cette « scénographie » précieuse, of. Myriam Dufour-Maître, Les Précieuses : naissance des femmes de lettres en France au XVIIe siècle, nouv. éd., Paris, Champion, 2008, p. 308.

${ }^{17}$ La présentation des lettres de Gas dans le Mercure galant prouve cependant que son apothéose était chose résolue et connue dès 1672: "Cette Dame en a fait le Cerbere du Parnasse, pour en défendre l'entrée aux mauvais Poëtes » (t. I, p. 268).

${ }^{18}$ Boileau, op. cit., pp. 134-135.

${ }^{19}$ Rappelons qu'il s'agit là des registres associés avec les grands satiriques latins : Juvénal (indignation et invective), Horace (enjouement et urbanité), Perse (érudition et morale).

20 «Les satires au féminin sont rarement reconnues comme telles, rarement lues comme telles. Parce qu'elles se cachent derrière tout un travail textuel qui, pour ingénieux qu'il soit, camoufle le tranchant de la charge sociale qu'il est censé mettre de l'avant. » (Lucie Joubert, « Hélène Monette : la satire au féminin », dans La Rhétorique au féminin, Annette Hayward (dir.), Québec, Nota Bene, 2006, p. 437). 
C’est ainsi que les idylles publiées dans le Mercure galant à partir de 1677, qui ont fait la réputation de Mme Deshoulières, commencent toutes par des exordes lyriques qui chantent le monde pastoral, innocent et paisible. Or, comme le souligne Sophie Tonolo, «L'utopie est un envers: elle s'élabore sur fond de satire ${ }^{21}$ ». La charmante peinture des "petits moutons » prépare un tableau bien différent, celui d'une réalité humaine qui est l'exact contraire d'un locus amoenus: "L'Ambition, l'Honneur, l'Intérêt, l'Imposture / Qui font tant de maux parmi nous, / $\mathrm{Ne}$ se rencontrent point chez vous » (p. 124). Alors que «Les Moutons » déplore les misères qui affligent l'homme en général, «Les Fleurs» et «Les Oiseaux» se livrent à une critique à peine voilée de la condition féminine : le pronom «nous » semble en effet référer ici d'abord et surtout aux femmes, maltraitées par les médisants, jaloux, ingrats et autres infidèles (pp. 170-171), et forcées par «l'usage, le devoir, l'austère bienséance » à «vivre dans la contrainte » (p. 189).

Sur le plan de l'énonciation, l'usage du «nous » contraste avec la posture typique du satiriste, moi hérö̈que et exceptionnel. Au lieu d'exhiber sa colère et d'attaquer directement des cibles humaines, le je lyrique s'adresse à la nature sur un ton plaintif et élégiaque. En effet, la franchise elle-même qui caractérise traditionnellement le verbe satirique semble être perdue dans le monde des hommes et passée entièrement de l'autre côté : «Il n'est de sincère langage, / Il n'est de liberté que chez les Animaux » (ibid.). En 1685, «Le Ruisseau » amplifie cette image d'une société où la parole de vérité a perdu toute vertu de correction morale. La locutrice déplore l'«Empire insolent» que l'homme «usurpe» sur la nature et les animaux, puis s'interrompt pour reconnaitre la vanité de son entreprise :

Ai-je quelque espoir de détruire

Des erreurs où nous nous plaisons?

Non, pour l'orgueil et pour les injustices

Le cœur humain semble être fait.

Tandis qu'on se pardonne aisément tous les vices,

On n'en peut souffrir le portrait.

Hélas! on n'a plus rien à craindre,

Les vices n'ont plus de Censeurs,

Le Monde n'est rempli que de lâches Flatteurs,

Savoir vivre, c'est savoir feindre. (p. 218)

Ces vers passent insensiblement du constat de l'inefficacité de la parole critique à celui de sa disparition totale, et d'une affirmation intemporelle sur «le cœur humain » à un diagnostic historique: «les vices n'ont plus de Censeurs ». Face à la corruption des mours, la nature apparait comme le dernier refuge de la vérité et de la liberté :

Ruisseau ce n'est plus que chez vous

Qu'on trouve encor de la franchise.

On y voit la laideur ou la beauté qu'en nous

La bizarre Nature a mise,

21 «Introduction », Poésies, p. 65. 
Aucun défaut ne s'y déguise,

Aux Rois comme aux Bergers vous les reprochez tous (ibid.) $)^{22}$.

De la même manière qu'elle avait délégué la poursuite des mauvais écrivains à son téméraire épagneul, Mme Deshoulières confie au ruisseau-miroir la tâche de démasquer les défauts humains. Le « fidèle cristal» est non seulement l'allocutaire mais le double du poète, son substitut naturel qui assume la mission devenue impossible dans la société.

Alors que les idylles offrent la nature comme contrepoint idéal au monde dépravé des hommes, d'autres poèmes de Mme Deshoulières assignent cette fonction au passé chevaleresque. C'est le cas surtout de la ballade, jadis célèbre, "À Caution tous Amants sont sujets » :

Une Pièce qui fit beaucoup de bruit, fut la Ballade qu'elle composa au mois de janvier 1684, sur le changement de la Cour en fait de galanterie, \& qu'elle adressa par une Épître au Duc de Montausier. L’Opéra d'Amadis venoit de paroître ; \& réveillant le souvenir des passions romanesques, qui ne subsistent plus que dans les Livres de Chevalerie, il avoit excité la bile de Madame Deshoulieres contre son siècle ${ }^{23}$.

Pour décharger sa «bile», l'auteur se sert une nouvelle fois d'un détour: au lieu d'écrire une satire régulière, elle compose une ballade en « vieux langage » à la manière de Voiture et de Sarasin. Le réquisitoire contre les mœurs contemporaines reste tranchant et amer, mais prend ici un tour enjoué et ludique :

Dans cœur humain probité plus n'habite ;

Trop bien encor a-t-on les mêmes dits

Qu'avant qu'Astuce au monde fût venue ;

Mais pour d'effets, la mode en est perdue ;

On n'aime plus comme on aimait jadis. (p. 136)

Le blâme du présent s'allie à la nostalgie du passé, de ce «Siècle d'Amadis » qu'il serait urgent de «ramener» (p. 137). Comme dans le cas de la «vieille chanson» d'Alceste (mais avec des valeurs différentes), le style de la ballade incarne lui-même l'idéal regretté : la galanterie spirituelle et tendre qui contraste avec l'indolence et l'insolence des «Jouvenceaux» et des «Amants grossiers». Si le temps des Amadis est révolu, du moins peut-on le faire revivre poétiquement, par la magie des mots. Le texte renvoie d'ailleurs à un modèle marotique précis, le rondeau «De l'Amour du Siecle Antique » dans lequel Marot lui-même regrettait, déjà, le « bon vieulx temps ${ }^{24}$ ».

${ }^{22}$ Le ruisseau rappelle le «canal» auquel La Fontaine compare le livre de La Rochefoucauld (Fables I, 11 : «L'Homme et son image »). Mme Deshoulières elle-même adressa un important poème à l'auteur des Maximes (« Ode à M.L.D.D.L.R. », dans Poésies, pp. 241-247).

23 "Éloge historique de Mme et de Mlle Deshoulières », dans CEuvres de Mme et de Mlle Deshoulières, Paris, chez les libraires associés, 1764, t. I, p. xxxiii. - Sur cette ballade et ses séquelles, voir Sophie Tonolo, "Aimer comme Amadis : Mme Deshoulières, une poétesse entre deux siècles ", dans Origines, Actes du 39e congrès de la NASSCFL, éd. Russell Ganim et Thomas M. Carr, Tübingen, Narr, 2009, pp. 275-285.

${ }_{24}$ Clément Marot, Euvres complètes, t. I, éd. François Rigolot, Paris, Garnier-Flammarion, 2007, p. 393. Pour la référence à Marot chez Mme Deshoulières, voir aussi son rondeau « Le bel Esprit, au siècle de Marot » (Poésies, pp. 172-173). 
Pour se protéger contre les inévitables ripostes masculines ${ }^{25}$, Mme Deshoulières compte sur le soutien d'autres hommes, figures d'autorité qui représentent l'équité et la vertu. C'est ainsi qu'elle invoque le jugement de Turgot de Saint-Clair, maître des Requêtes, qui semble avoir cautionné sa condamnation des «peu tendres amours »:

Par là, d'une orgueilleuse et mordante Critique,

Je ne sentirai point le dangereux pouvoir.

Oui, puisque vous louez l'horreur que je fais voir

Des vices où le siècle abonde,

On n'osera blâmer mon juste emportement. (p. 282)

Et quand Mme Deshoulières assemble le premier volume de ses Poésies (paru en 1688), elle introduit la ballade par une épitre au vieux duc de Montausier:

MONTAUSIER dont le cœur ferme, grand, et sincère

Seul dans un siècle corrompu

Possède, connait, et révère

Le vrai mérite, et l'antique vertu :

Souffrez qu'en vos mains je dépose

Les innocents chagrins de mon cœur irrité. (p. 134)

Soupirant puis époux de Julie d'Angennes, traducteur des Satires de Perse, gouverneur du Dauphin, modèle présumé de l'Alceste de Molière, Montausier est le lecteur idéal à qui Mme Deshoulières livre «les ennuis profonds / Que souffre un cœur fidèle et tendre, / Dans un siècle où l'Amour n'est que dans les chansons » (p. 135).

Ces « innocents chagrins » confiés à Montausier nous amènent pour finir à la série des Épitres chagrines dans lesquelles Mme Deshoulières approche le plus de la satire régulière. Aussi est-ce par une de ces épîtres qu'elle est représentée dans l'anthologie des Satires du XVII siècle éditée par Fernand Fleuret et Louis Perceau, seule femme à côté de 56 satiriques masculins ${ }^{26}$. Elle emprunte l'appellation générique à Scarron, qui composa entre 1652 et 1660 quatre Épitres chagrines, dont la dernière est d'ailleurs adressée à l'auteur de la Clélie. Le mot « chagrin » dénote ici aussi bien la tristesse que l'irritation, l'humeur à la fois fâchée et fâcheuse. Un événement déplaisant (le départ d'un ami, la visite d'un fou) agace et ennuie le poète, qui se met à pester contre tous ceux qui l'enragent - beaux esprits, pédants, précieuses, le monde entier enfin - allant jusqu'à vouloir s'exiler: "Il faut porter dans l'Amerique / Un chagrin si melancolique $^{27} » \ldots$ Bien que ces textes abondent en motifs et traits satiriques, le style particulier de Scarron, facétieux et désinvolte, évite la violence de l'invective juvénalienne et «raille sans acrimonie ${ }^{28} »$. Cette manière légèrement décalée d'écrire

25 Voir surtout la ballade que La Fontaine composa sur les mêmes rimes, peignant Mme Deshoulières en "femme un peu trop dépite ", "Dame chagrine » dont l' " humeur bourrue » doit être l'effet d'une " déconvenue » sentimentale (CEuvres diverses, éd. Pierre Clarac, Paris, Gallimard, 1958, pp. 622-623 ; ce poème ne fut pas imprimé du vivant de La Fontaine et parut pour la première fois en 1747 dans les (Euvres de Pavillon).

26 T. II, Paris, Garnier, 1923, pp. 199-207.

27 Poésies diverses, éd. Maurice Cauchie, t. II, Paris, Didier, 1960, p. 65.

${ }^{28}$ Marcel Simon, «Les Épîtres chagrines de Scarron », Littératures classiques, no 18, 1993, p. 183. 
la satire, de l'intégrer dans un cadre épistolaire et de la lier étroitement à l'humeur et la sensibilité du poète avait de quoi plaire à Mme Deshoulières, qui reprit le modèle scarronien à sa façon.

Or, ce qui provoque le chagrin de l'épistolière, c'est surtout la parole - ignorante, insolente, hypocrite - des autres. Ainsi, le premier de ces poèmes avertit Mlle de La Force (future romancière et conteuse) des "dégoûts» que ressent un «bel Esprit» dans la société contemporaine, «sitôt que par la voix commune / De ce titre odieux on se trouve chargé » (p. 128). À la ville comme à la Cour, être femme (ou homme) de lettres signifie s'exposer à des conversations insipides, des interrogatoires importuns, des jugements présomptueux :

Un esprit de malignité

Dans le monde a su se répandre.

On achète un bon Livre, afin de s'en moquer (p. 129).

La deuxième épitre (nouvelle récrimination contre le déclin de la galanterie) commence par la tirade abrupte d'un jeune «Fat» qui accoste l'épistolière, pendant que celle-ci reste murée dans un silence songeur:

Quel discours pouvais-je lui faire,

Moi qui dans ce même moment

Repassais dans ma tête avec étonnement

De la nouvelle Cour la conduite ordinaire?

M'aurait-il jamais pardonné

La peinture vive et sincère

De cent vices auxquels il s'est abandonné ? (pp. 180-181)

Au lieu d'être présentée de vive voix aux courtisans eux-mêmes (opération dangereuse et inutile), la peinture des vices de la cour sera donc confiée au papier, sous la forme d'une épître envoyée à une amie de province.

Cette structure énonciative se retrouve dans la troisième épitre, adressée au Père de La Chaise. Elle aussi s'ouvre sur la transcription, au discours direct, des propos de sa cible, un faux dévot «d'un nom que par raison je tais» (p. 312). Horrifiée par sa casuistique scandaleuse, l'épistolière entreprend de sermonner le «Scélérat» mais celui-ci la quitte brusquement, "tandis qu'en discours ma colère s'exhale » (p. 314). Ici encore, c'est donc par écrit que l'auteur décharge sa bile et dénigre les hypocrites, menaçant même, si la calomnie l'y force, d'appeler un chat un chat :

Et s'ils osaient sur moi répandre leur noirceur, Quelque Ouvrage pourrait paraittre,

Où je les traiterais avec moins de douceur, Et par leurs noms enfin je les ferais connaittre. (p. 316)

Alors que «la peinture vive et sincère » des vices n'est plus admise dans le commerce social et que la parole franche a perdu toute efficacité, l'écriture au moins peut encore 
remplir cette mission de censure publique. Les «chagrins » causés par le spectacle du monde commencent par réduire l'écrivain au silence, pour s'exprimer ensuite par le détour de la littérature.

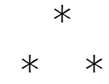

Quelques mois avant sa mort, Mme Deshoulières se fit peindre par ÉlisabethSophie Chéron et publia à cette occasion des Réflexions morales sur l'envie immodérée de faire passer son nom à la postérité. Elle y raille le «fol orgueil » des hommes et critique le «faux bien» de la gloire posthume, avant de s'interpeller et de se reprendre ellemême :

Mais d'où vient qu'aujourd'hui mon esprit est si vain?

Que fais-je ! et de quel droit est-ce que je censure

Le goût de tout le genre humain [...] ?

Ai-je acquis dans le monde assez d'Autorité

Pour rendre mes raisons utiles,

Et pour détruire en lui ce fonds de vanité

Qui ne lui peut laisser aucuns moments tranquilles?

Non, mais un esprit d'équité

À combattre le faux incessamment m'attache,

Et fait qu'à tout hasard j'écris ce que m'arrache

La force de la vérité (p. 344).

« Non, mais... »: même au terme de sa carrière, l'auteur n'a toujours pas acquis assez d'«Autorité », et pourtant elle persiste à écrire et à combattre, à tout hasard. En défendant ainsi la légitimité de sa vocation poétique, Mme Deshoulières s'approprie une dernière fois un élément traditionnel du genre lucilien, l'apologia pro satura sua dans laquelle le satiriste répond aux objections faites contre son audacieuse entreprise. Ses vers préfigurent l'autoportrait apologétique que Boileau, très soucieux (surtout après le scandale de la "Satire $\mathrm{X}$ ») de faire passer une bonne image de lui-même à la postérité, dressa en 1695 dans son « Epître X» :

Déposez hardiment qu'au fond cet homme horrible,

Ce censeur qu'ils ont peint si noir et si terrible,

Fut un esprit doux, simple, ami de l'équité,

Qui, cherchant dans ses vers la seule vérité,

Fit, sans être malin, ses plus grandes malices :

Et qu'enfin sa candeur seule a fait tous ses vices ${ }^{29}$.

${ }^{29}$ Boileau, op. cit., p. 212. À ce sujet, voir mon article «Classique par anticipation: Boileau et le fol espoir de l'immortalité », Euvres \& Critiques XXXVII, 1 (2012), pp. 125-141. - La «candeur» que Boileau associe avec la satire (voir aussi Art poétique III, 179) apparaît chez Mme Deshoulières dans une épître à Mme de Maintenon : «Loin de savoir flatter, apprends que je me pique / De cette candeur héroïque / Qu’au nombre des vertus on recevait jadis» (Poésies, p. 210). 
Équité - vérité: la rime porterait-elle la trace de Mme Deshoulières ? Son fantôme hanterait-il cette apologie ? Cela peut paraitre invraisemblable, mais je ne m'interdis pas d'imaginer, à tout hasard, que cet écho poétique recèle un secret hommage de Boileau à son antagoniste, une reconnaissance tardive de ce qui reliait, malgré leurs différences, ces deux spirituels « censeurs » de leur siècle.

Volker SCHRÖDER

Princeton University 